\title{
Dynamic Monte-Carlo modeling of hydrogen isotope diffusion in co-deposited layers
}

\author{
A. Rai ${ }^{* a}$, Maya P. N. ${ }^{b}$, R. Schneider ${ }^{a}$, S. P. Deshpande ${ }^{b}$, M. Warrier $^{b}$ \\ ${ }^{a}$ Max-Planck-Institut für Plasmaphysik, Wendelsteinstrasse 1, D-17491 Greifswald, Germany \\ ${ }^{b}$ Institute for Plasma Research, BHAT, Gandhinagar, Gujarat, India, 382428
}

\begin{abstract}
Reactive-diffusive transport of hydrogen in the porous, 3-dimensional structure of codeposited films is simulated using a kinetic Monte Carlo (KMC) method. The simulations are carried out on samples generated from the film growth Monte Carlo (FGMC) code which simulates the film-growth process in remote regions of fusion devices. It is seen that the steric repulsion between the incoming hydrocarbon and the surface atoms is an important parameter which affects the structure and trap concentrations of these soft films. Initial results indicate that diffusion of hydrogen through pores in the co-deposited films could be a major contributor to the high density of hydrogen in co-deposits seen at remote locations of fusion devices.
\end{abstract}

JNM keywords: Carbon, Diffusion, Hydrogen, Theory and Modeling.

PSI-17 keywords: Carbon impurities, Co-deposition, Hydrocarbons, Hydrogen Inventory, Erosion and Deposition.

PACS codes: 68.55.-a, 68.55.AC, 66.30.-h, 66.30.Jt, 66.30.Ny

*Corresponding author address and e-mail: Stellaratortheorie, Max-Planck-Institut für Plasmaphysik, Wendelsteinstrasse 1, D-17491 Greifswald, Germany; Abha.Rai@ipp.mpg.de Presenting author name and e-mail: Manoj Warrier; manoj.warrier@ipp.mpg.de 


\section{Introduction}

Graphite or carbon fiber composites (CFC) are used extensively as plasma facing components (PFC) in magnetic confinement based fusion devices. Erosion of carbon [1-4] as hydrocarbons and its co-deposition form hydrocarbon films on the inner surface of these devices. The deposits are porous and can form both hard and soft films [5-8]. The hard films are found in regions of the fusion device where annealing of the deposits is possible due to energetic particle incidence. The soft films are found in remote regions of the device where energetic particles are not present or where the temperature of the surface is not high enough to anneal the films $[9,10]$. The hard films contain typically $\mathrm{H}$ concentrations of a few percent of the carbon concentration, and its extent is limited to a depth of $1 \mu \mathrm{m}$. The soft films on the other hand, contain hydrogen concentrations of around 70-100 percent of the carbon concentration and are present throughout the depth of the co-deposited layer, which is typically several tens of $\mu m[9,10]$.

It is expected that these soft deposits at remote locations will be one of the major channels of tritium retention in fusion reactors [11]. The co-deposited films can also affect the hydrogen wall inventory in steady state machines [12]. Understanding the underlying processes of co-deposited layer formation and hydrogen retention in such soft films, requires not only experimental characterization of the structure and composition of such layers as done in $[5,9,10]$ but also multi-scale models which link the atomistic interactions to macroscopic growth models. In this paper we simulate the reactive-diffusive transport of 
hydrogen isotopes in such layers. The model for the co-deposited layer has been briefly described here in section 2 (for details see [13]).

The transport simulation has been carried out by extending the previously developed multi-scale model for hydrogen isotope diffusion and reactive-diffusive processes in porous graphite $[14,15]$. It is seen from this simulation that atomic hydrogen diffusion through the porous co-deposits and subsequent trapping at trap sites can be a major contribution to the high density of hydrogen in these soft films.

Section 3 gives the details of the reaction-diffusion model and the simulation. In the next section we present the results of the reactive-diffusive simulation in co-deposits of different porosities. Finally we present our conclusions and outlook.

\section{Monte-Carlo simulation of film growth at remote regions}

\subsection{Model}

In this model a graphite surface having a specified initial population of dangling bonds is considered to be in contact with a vapor phase consisting of a mixture of fuel-gas neutral atoms and methyl radicals. The key ingredient of the model which distinguishes it from the conventional ballistic models for film growth [16] is the shape and structure of the molecule. We follow a Monte-Carlo (MC) ansatz in which particles can arrive at any angle 
and stick upon first contact with a dangling bond. The choice of target sites are made by considering shadowing effects described in detail in [13]. Various events are possible at a chosen site depending on the local atomic neighborhood. The events considered are:

(1) H-abstraction due to incident $\mathrm{CH}_{3}$ with a probability $\mathrm{P}_{a b s}$ at sites having a $\mathrm{H}$ atom,

(2) bonding of $\mathrm{CH}_{3}$ along with $\mathrm{H}$-abstraction ( $\mathrm{sp}^{2}$ type) at sites having a dangling bond,

(3) bonding of the methyl radical with a dangling bond ( $\mathrm{sp}^{3}$ type) and

(4) null event which can happen at any site.

The a-C:H network formed in this way is more complex due to the presence of two hybridization states, $\mathrm{sp}^{2}$ and $\mathrm{sp}^{3}$, of carbon. In addition, the growth at a particular location can be terminated if the H-atom there is not removable. The orientation of the molecule being attached is decided by a valance shell electron pair repulsion (VSEPR) like ansatz [17], since this is computationally much cheaper to implement than $\mathrm{MC}$ or molecular dynamics (MD) relaxation. Then, subject to the the interatomic distance between a $\mathrm{H}$ atom of the attached molecule and a $\mathrm{H}$ atom on the target being greater than a critical value, $d_{c r i t}$, the possibility of either $\mathrm{sp}^{2}$ or $\mathrm{sp}^{3}$ type of bonding event is explored. $d_{\text {crit }}$ is an indirect measure of the steric repulsion between the incoming hydrocarbon and the surface atoms of the target, and was found to be the most important parameter affecting the micro-porosity and surface roughness of the films [13]. Hydrocarbon films of different structure and trap site distributions have been generated by using different values of the parameter $d_{c r i t}$. If both are possible, then a selection is made using the probability $P_{s p 23}$. If no meaningful event is possible the site is considered as blocked. An event-list is thus created by exploring both $\mathrm{sp}^{2}$ and $\mathrm{sp}^{3}$ types of bonding. 


\subsection{Different structures generated by the model}

Using the above model a-C:H films were grown on square sample of size $56 \times 56 \AA^{2}$. Periodic boundary conditions were implemented along $\mathrm{X}$ and $\mathrm{Y}$ directions, which form the plane of the surface on which the co-deposited film is grown. A typical film grown by the FGMC code is shown in Fig.1. Other inputs are, $P_{a b s}=0.9, P_{s p 23}=0.5$ and initial surface density of methyl radicals, $\mathrm{rad}=0.1$, corresponds for $10^{15} / \mathrm{cm}^{2}$, which can be considered as a good estimate of typical areal density of dangling bonds [18]. Multiple showers of methyl radicals were considered to grow films of thickness $\sim 70-100 \AA$. Table.1 shows the variation of density, porosity, ratio of number of hydrogen atoms to carbon atoms $(\mathrm{H} / \mathrm{C})$ and a similar ratio for the number of traps $\left(n_{\text {trap }} / \mathrm{C}\right)$ as a function of $d_{\text {crit }}$ before starting the reactive-diffusive process. Note that the density (porosity) of the film decreases (increases) with $d_{\text {crit }}$. The number of traps for $d_{\text {crit }}=2.0$ and $d_{\text {crit }}=3.0$ are statistically almost the same (12487 and 12316 respectively) compared to the number of traps for the $d_{c r i t}=1.5$ case $(9822)$. The contribution of voids of different sizes to the porosity is shown as a function of $d_{c r i t}$ in Fig.2. Note that the peak of the void-size distribution shifts from voids of size $5 \AA$ to $10 \AA$ as $d_{\text {crit }}$ varies from 1.5 to 3.0. Hydrogen transport in such a film would depend on the porosity, void sizes and number of traps in such films. It is therefore interesting to study the diffusion and trapping of hydrogen atoms in such porous films from a hydrogen inventory point of view. 
Table 1

Variation of co-deposited film parameters with $d_{\text {crit }}$

\begin{tabular}{|c|c|c|c|c|}
\hline $\begin{array}{c}d_{\text {crit }} \\
(\AA)\end{array}$ & $\begin{array}{c}\text { density } \\
(\mathrm{gm} / \mathrm{cc})\end{array}$ & $\begin{array}{c}\text { porosity } \\
(\text { volume fraction })\end{array}$ & $\mathrm{H} / \mathrm{C}$ & $n_{\text {trap }} / \mathrm{C}$ \\
\hline 1.5 & 1.681 & 0.331 & 0.187 & 0.546 \\
\hline 2.0 & 1.111 & 0.432 & 0.144 & 0.812 \\
\hline 3.0 & 0.960 & 0.480 & 0.119 & 0.903 \\
\hline
\end{tabular}

\section{Reaction-diffusion model for transport in porous media}

In the earlier work hydrogen isotope diffusion in porous graphite was modeled using a time dependent, 3-dimensional, multi-scale model $[14,19,20]$. The porous geometry was constructed using statistical distributions for crystallite dimensions and crystallite orientations for a specified micro-void fraction. The model used molecular dynamics (MD) at the micro-scales $\left(2.5 \mathrm{~nm}, 10^{-10} \mathrm{~s}\right)$ and consistently parametrized the MD results within a KMC scheme [19]. The KMC scheme was extended to include trapping and detrapping at the crystallite-micro-void interface to simulate trans-granular-diffusion (TGD) at the meso-scales $\left(10^{-7}-10^{-6} \mathrm{~m}\right.$, several $\left.\mathrm{ms}\right)$ [20] using the results from our micro-scale modeling and from experiments ([21] and references therein). This concept was then extended to the macro-scales (1 cm, up to a few seconds), by using the parametrized TGD diffusion co-efficient in a Monte Carlo diffusion model with transport in voids using a KMC model, thereby having a truly multi-scale capability [14].

The TGD version of the above multi-scale model was modified to include reactions by specifying a Smoluchowski boundary condition [22] for species recombination, wherein 
the species wandering a certain distance $r_{o}$ of each other recombine. This is equivalent to having a step potential well for interaction of the species at a radial distance $r_{o}$ from the center of the interacting specie. Such a boundary condition is valid for cases with strong binding energies (like hydrogen recombination or hydrogen trapped at a open bond site) where reflection becomes a rare event once the atom gets closer than a cutoff distance [23]. Initial results for hydrogen re-emission from graphite using the modified code match experiments both qualitatively and quantitatively [15]. In the present work the code has been used to simulate the transport of hydrogen in the porous co-deposits and the trapping of hydrogen at open bond sites.

The reactive-diffusive transport of hydrogen has been simulated in the co-deposits at 300 $\mathrm{K}$ and at $500 \mathrm{~K}$ so as to represent typical temperatures of the surfaces in remote locations. In reality, the migration energy of the hydrogen in the internal surfaces of such a film will depend upon the local composition and hybridization states of carbon atoms. Due to lack of data (experimental or MD simulation), the migration energy for surface diffusion has been chosen to be $0.5 \mathrm{eV}$, which is of the same order as the binding energy of hydrogen bonds and of hydrogen surface diffusion in graphite [24]. The jump lengths are taken to be of the order of surface diffusion jump lengths in graphite $(\sim 30 \AA)$ [20], for a similar reason as above. This does not affect the results as the size of the jumps is actually limited by the pore size of the co-deposits which have a distribution as shown in Fig.2. 


\section{Results from the reactive-diffusive transport simulation in co-deposited layers}

Based on the size of the co-deposited sample generated by the FGMC code, 400 atoms of hydrogen were initialized randomly on the surface of the film. This corresponds to an average interatomic distance just greater than 2 A. Fig. 3 shows the trapping of hydrogen atoms at the trap sites within the co-deposits at $300 \mathrm{~K}$. The trend is exactly similar in the $500 \mathrm{~K}$ case, except for the time taken for trapping. It is seen that $60-65 \%$ of the hydrogen atoms are trapped and the rest are desorbed as hydrogen molecules. It is seen that the fraction trapped depends on the number of trap sites, which is why the fraction trapped in the $d_{\text {crit }}=1.5$ case $(60 \%$ trapped $)$ is smaller than the fraction trapped in the $d_{\text {crit }}=2.0$ and $d_{\text {crit }}=3.0$ cases $(\sim 65 \%$ trapped $)$. It is expected that at larger fluence, most of the trap sites in the co-deposited film get filled by the diffusing hydrogen atoms. The hydrogen concentration in such films can match the values reported in experiments for co-deposits at remote locations $[9,10]$. The effect of temperature seems to be mainly to speed up the trapping process. The time scale of trapping is around 0.1 milli-secs at $300 \mathrm{~K}$ and less than a tenth of a micro-sec at $500 \mathrm{~K}$.

\section{$5 \quad$ Summary and future directions}

Co-deposited film formation at remote locations has been simulated. Initial results for hydrogen diffusion and trapping in these films are presented. It is seen that a large fraction (60-65 \%) of the incident hydrogen atoms are trapped at trap locations of the co-deposited 
film and this could be a major contributor to the high density of hydrogen in co-deposits at remote locations of fusion devices.

Extensions of the reaction-diffusion code to include realistic fluxes will help in better quantifying the hydrogen trapping in co-deposited layers. For example, as the trap sites get filled, the hydrogen atoms will have to diffuse further into the film and the time scale of trapping will increase. Quantifying such effects require changes in the reactive-diffusion model to handle steady state fluxes, which is already being done. The migration energies used in this simulation are expected to be of the right order, but confirmation of this by molecular dynamics simulations (as in [19]) or by experiments on hydrogen diffusion in co-deposits is required. The molecular dynamics simulations to generate the migration energies as a function of the hydrogen content of the films is part of future work. More interactions in FGMC with more species of incident hydrocarbons, along with simulated annealing using realistic interaction potentials is desirable, which will allow modeling of hard co-deposits.

\section{Acknowledgment}

R. Schneider and A. Rai acknowledge funding of the work by the Initiative and Networking Fund of the Helmholtz Association. M. Warrier acknowledges funding from the MaxPlanck India Fellowship. 


\section{References}

[1] J. Küppers. Surface Science Reports, 22, (1995) 249.

[2] J. Roth. J. Nucl. Mater., 266-269, (1999) 51.

[3] E. Salonen, K. Nordlund, J. Keinonen, and C. H. Wu. Phys. Rev. B, 63, (2001) 195415.

[4] C. Hopf, A. von Keudell, and W. Jacob. Jnl. App. Phys., 94 (4), (2003) 2373.

[5] Pascale Roubin. J. Nucl. Mater., 337-339, (2005) 990.

[6] V. N. Denisov, L. A. Kuzik, N. Lvova, et. al. Physics Letters, 239(4-5), (1998) 328.

[7] M. Mayer, V. Rohde, A. von Keudell, and ASDEX Upgrade Team. J. Nucl. Mater., 313-316, (2003) 429 .

[8] W. Jacob. J. Nucl. Mater., 337-339, (2005) 839.

[9] J. von Seggern, A. Kirschner, V. Philipps, and P. Weinhold. Physica Scripta, T111, (2004) 118.

[10] M. J. Rubel, J. P. Coad, P. Weinhold, and G. Matthews et. al. Physica Scripta, T111, (2004) 112.

[11] G. Federici et. al. Nuclear Fusion, 41(12R), (2001) 1967.

[12] E. Tsitrone, C. Brosset, and J. Bucalossi et. al. Proceedings, 20 ${ }^{\text {th }}$ IAEA Fusion Energy Conference, Vilamoura, Portugal, 1-6 Nov., IAEA-CN-116/EX/10-2, (2004).

[13] Maya P. N, S. P. Deshpande, and M. Warrier. $10^{\text {th }}$ Int. Workshop on Plasma Edge Theory on fusion devices, Jülich, Germany, 17-19 Oct. 2005, Accepted for publication, Contrib. Plasma. Phys., (2006). 
[14] M. Warrier, R. Schneider, E. Salonen, and K. Nordlund. Jnl. Nucl. Mater., 337-339, (2005) 580.

[15] R. Schneider, A. Rai, A. Mutzke, M. Warrier, et. al. 12th Int. Conference on Fusion Reactor Materials, Dec 4-9, Santa Barbera, California, USA, Accepted for publication, Jnl. Nucl. Mater., (2006).

[16] P. Meakin, P. Ramanlal, L. Sander, and R. Ball. Physical Review A, 34(9) (1986) 152.

[17] R. J. Gillespie. Molecular Geometry, Van Nostrand Reihold, Londres., (1972).

[18] W. Möller, W. Fukarek, K. Lange, et. al. Jpn. J. Appl. Phys., 34, (1995) 2163.

[19] M. Warrier, R. Schneider, E. Salonen, and K. Nordlund. Physica Scripta, T108 (2004) 85.

[20] M. Warrier, R. Schneider, E. Salonen, and K. Nordlund. Contrib. Plasma Phys., 44(1-3), (2004) 307.

[21] K. L. Wilson et. al. Atomic and plasma-material interaction data for fusion (supplement to the journal Nuclear Fusion, 1, (1991) 31.

[22] M. V. Smoluchowski. Z. physik. Chem., 92, (1917) 192.

[23] T. R. Waite. Physical Review, 107(2), (1957) 463.

[24] R. A. Causey, M. I. Baskes, and K. L. Wilson. J. Vac. Sci. Technol. A, 4(3), (1986) 1189. 


\section{List of Figures}

1 A typical film grown by the film growth Monte-Carlo (FGMC) code. The picture on the left is a wire-frame view which emphasises the bonding chains and the picture on the right is a view with atoms and bonds. The direction of growth is indicated by the arrow. The hydrocarbon atoms that form the film are incident in a downward direction

2 Variation of the void size contribution to total porosity as a function of void size (in units of number of $1 \AA$ sized bins) for different values of the steric repulsion parameter, $d_{\text {crit }}$

3 Fraction of hydrogen trapped as a function of time at $300 \mathrm{~K}$ for different values of $d_{\text {crit }}$ 


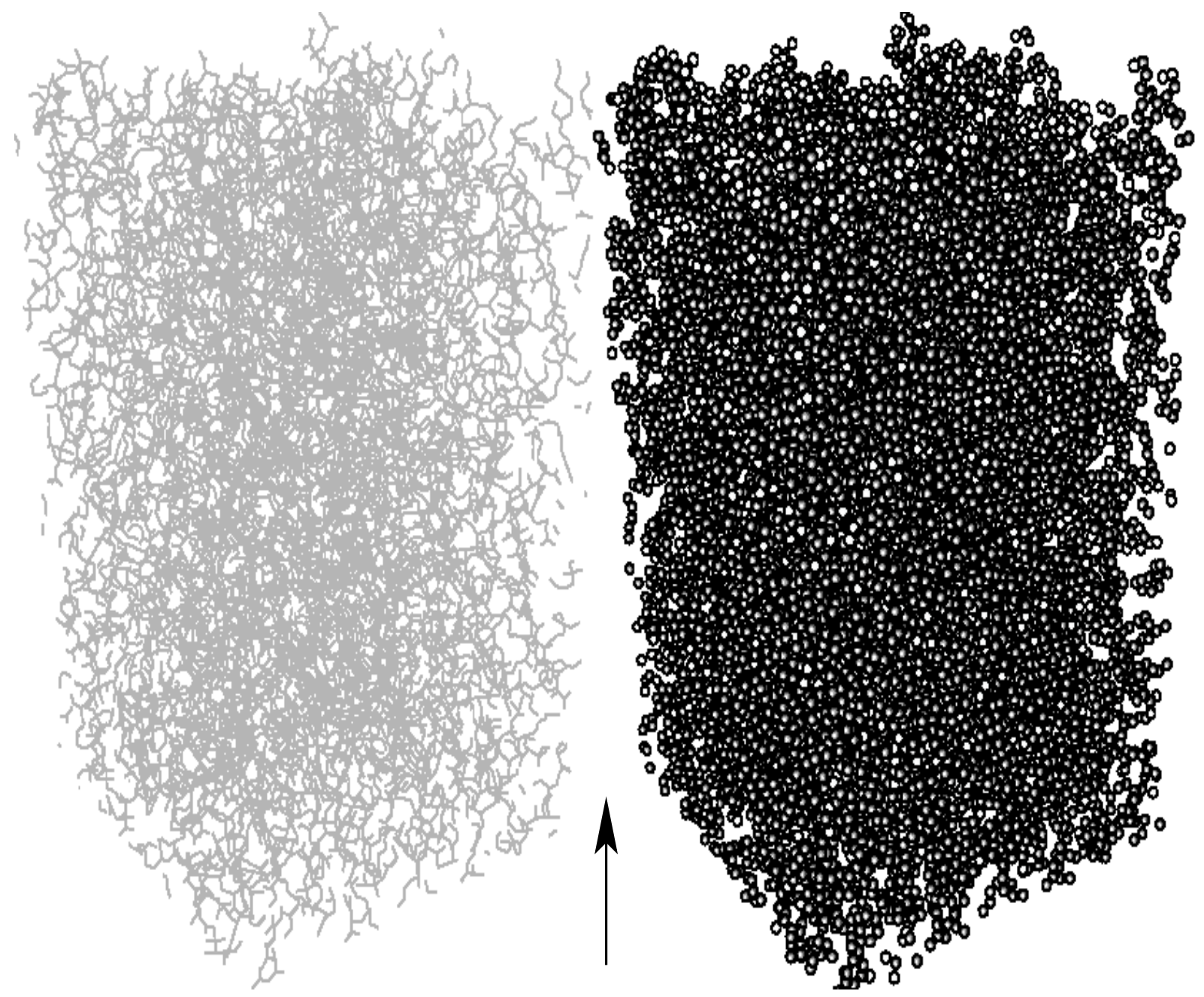

Fig. 1. A typical film grown by the film growth Monte-Carlo (FGMC) code. The picture on the left is a wire-frame view which emphasises the bonding chains and the picture on the right is a view with atoms and bonds. The direction of growth is indicated by the arrow. The hydrocarbon atoms that form the film are incident in a downward direction 


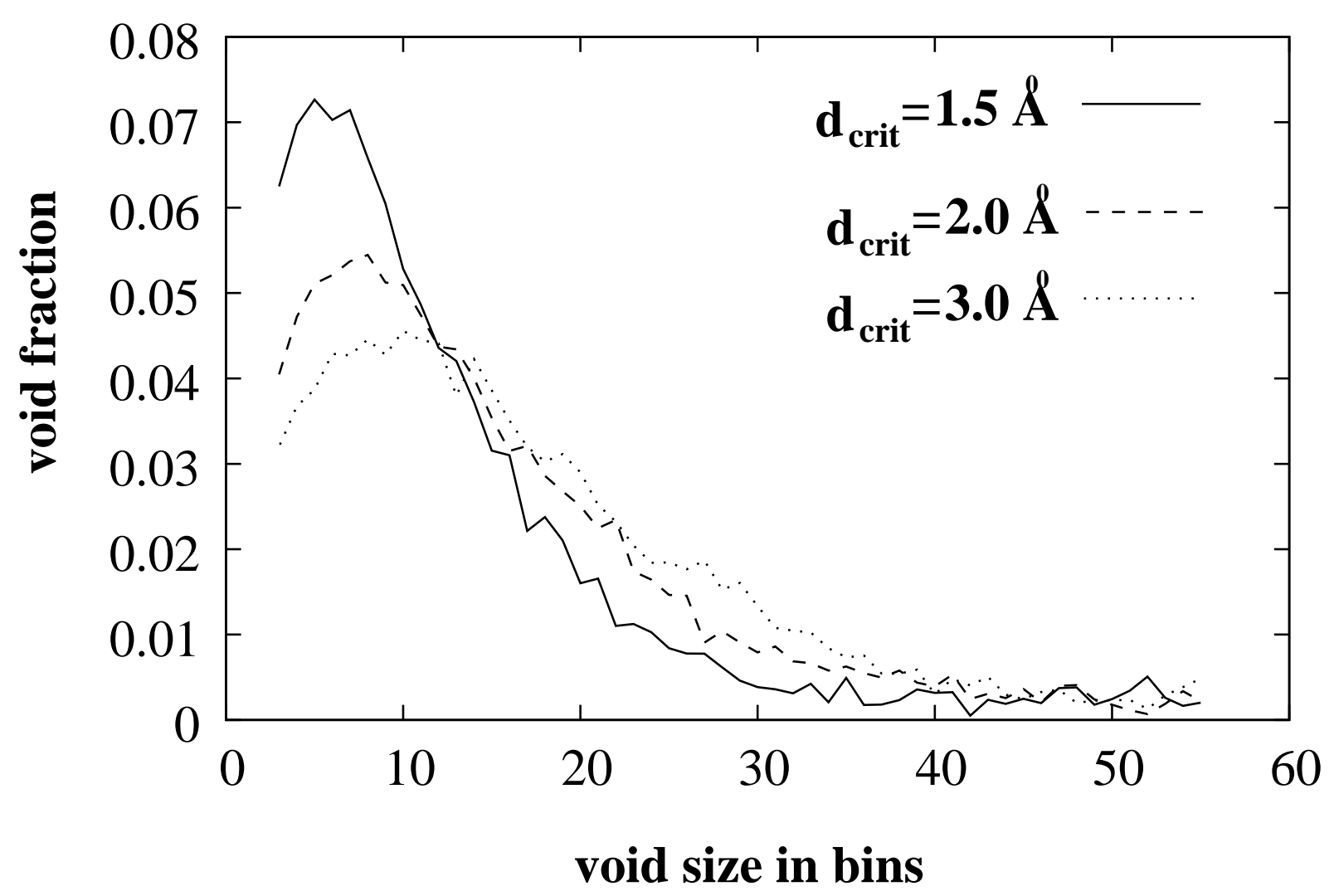

Fig. 2. Variation of the void size contribution to total porosity as a function of void size (in units of number of $1 \AA$ sized bins) for different values of the steric repulsion parameter, $d_{c r i t}$ 


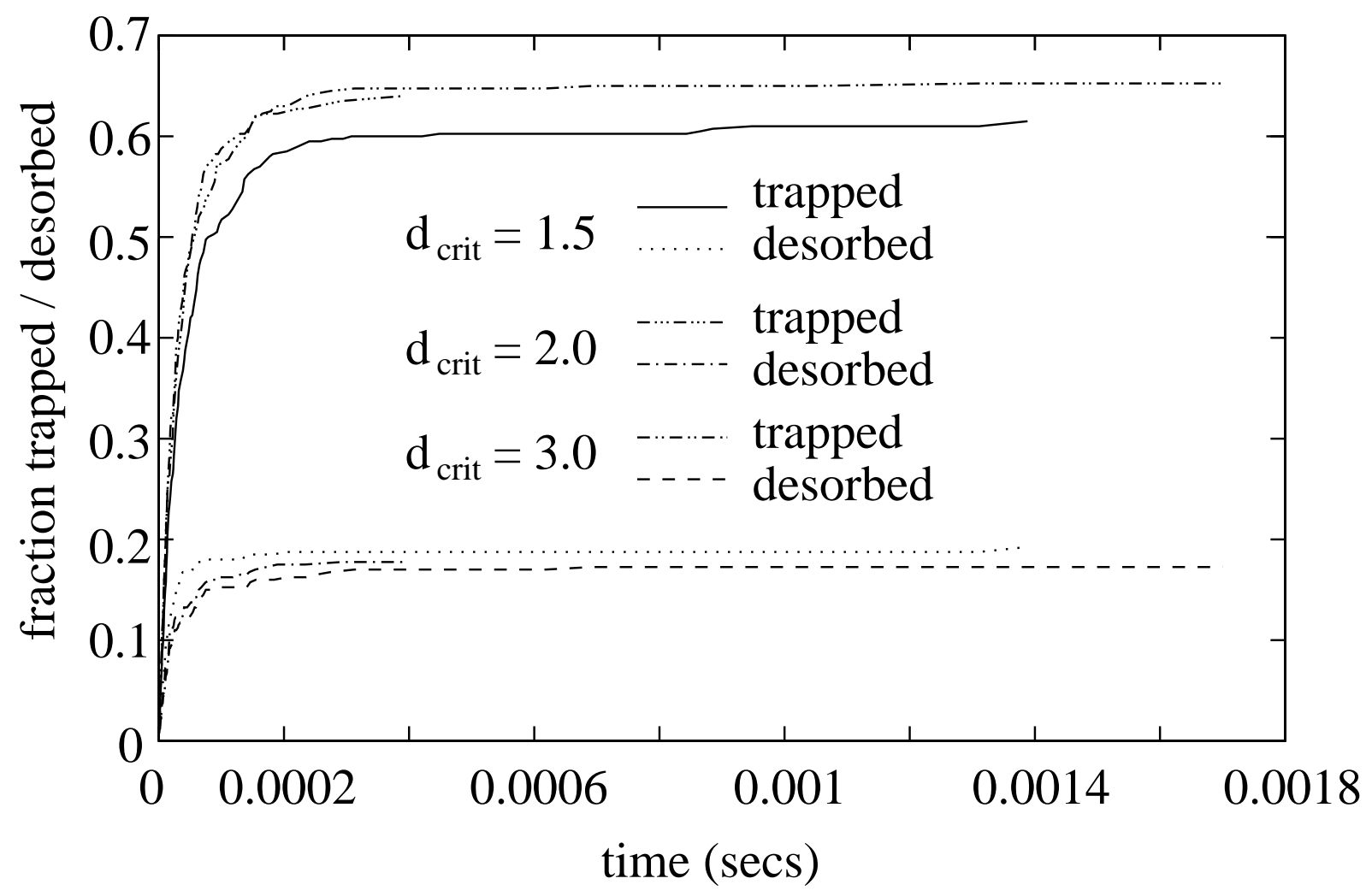

Fig. 3. Fraction of hydrogen trapped as a function of time at $300 \mathrm{~K}$ for different values of $d_{\text {crit }}$ 